\title{
Operations analysis of behavioral observation procedures: a taxonomy for modeling in an expert training system
}

\author{
Roger D. Ray • Jessica M. Ray • David A. Eckerman • \\ Laura M. Milkosky • LaDarien J. Gillins
}

Published online: 30 July 2011

(C) Psychonomic Society, Inc. 2011

\begin{abstract}
This article introduces a taxonomy based on a procedural operations analysis (Verplanck, 1996) of various method descriptions found in the behavior observation research literature. How these alternative procedures impact the recording and subsequent analysis of behavioral events on the basis of the type of time and behavior recordings made is also discussed. The taxonomy was generated as a foundation for the continuing development of an expert training system called Train-to-Code (TTC; J. M. Ray \& Ray, (Behavior Research Methods 40:673-693, 2008)). Presently in its second version, TTC V2.0 is software designed for errorless training (Terrace, (Journal of the Experimental Analysis of Behavior 6:1-27, 1963)) of student accuracy and fluency in the direct observation and coding of behavioral or verbal events depicted via digital video. Two of 16 alternative procedures classified by the taxonomy are presently modeled in TTC's structural interface and functional services. These two models are presented as illustrations of how the taxonomy guides
\end{abstract}

Electronic supplementary material The online version of this article (doi:10.3758/s13428-011-0140-6) contains supplementary material, which is available to authorized users.

R. D. Ray $(\bowtie) \cdot J$. M. Ray $\cdot$ L. M. Milkosky $\cdot$ L. J. Gillins

Rollins College,

Winter Park, FL, USA

e-mail: rdray@rollins.edu

J. M. Ray

University of Central Florida,

Orlando, FL, USA

D. A. Eckerman

University of North Carolina at Chapel Hill,

Chapel Hill, NC, USA software user interface and algorithm development. The remaining 14 procedures are described in sufficient operational detail to allow similar model-oriented translation.

Keywords Behavioral observation - Observation procedures - Observer training - Computer based training . Observer agreement $\cdot$ Expert training systems

In most descriptive research literatures, observers use linguistic labels to record an account of ongoing behaviors, be they discerned by auditory, visual, or any other sensory channel (see Bakeman \& Gottman, 1987,1997; Martin \& Bateson, 1993; Sharpe \& Koperwas, 2003; Wallace \& Ross, 2006). Observers are trained to classify, or code, behavior by appropriate application of a linguistic category, whether for data gathering, behavioral assessment, or intervention/treatment purposes (see Friman, 2009). The degree to which training has been successful is normally measured through statistical techniques for assessing interobserver agreement (IOA) or intraobserver agreement-techniques such as percentage of agreement, Cohen's kappa, correlations, ratios of frequency or duration estimates, as well as others (see Bakeman \& Gottman, 1997; Hartmann, 1977; Mitchell, 1979; Wallace \& Ross, 2006).

There is a large variation in experimental procedures for carrying out behavioral observation and recording, and these procedures have a variety of descriptive summary labels that have been used in the literature. In this article, we reconstruct from published procedural descriptions each of the types of procedures used-an effort that W. S. Verplanck (1957, 1996) called operations analysis. In Verplanck's (1957) earliest definition of this approach, he empirically established the exact meaning of scientific terms used by various researchers and published his earliest 
version as $A$ Glossary of Terms. ${ }^{1}$ We follow this tradition and offer a more consistent and procedurally based terminology, or verbal taxonomy, for classifying the various observation procedures to be found in the literature. This not only offers utility by establishing a common vocabulary for discussing widely varying systematic observation procedures, but also offers the prospect of specifying functional and structural requirements of computer interfaces and algorithms designed to aid in data collection using any given procedure. This computerized design process represents at least one form of modeling such procedures (i.e., constructing structural and functional exemplars for them).

This article also provides operational specifications that would allow us to extend an expert software system, previously described in this journal (J. M. Ray \& Ray, 2008), that models only two of the existing procedures reported in the behavior observation research literature. This system, called train-to-code ${ }^{2}$ (TTC), is a comprehensive program for training behavioral observation/recording repertories using errorless training strategies (Terrace, 1963). A secondary purpose of this article is to present an introductory review of the issues underlying observation research in general. A systematic survey of literature conducted for this effort found few discussions that have compared and contrasted the many problems, issues, decisions, and implications that should be considered before selecting an observational procedure. Thus, we provide a procedure-byprocedure guide through these considerations.

The first type of procedure modeled in TTC was a continuous coding procedure adequate for serving as an expert coding reference. In TTC, Continuous Coding provides a frame-by-frame classification of any incorporated video source and is thus sufficient to provide feedback and correction for any trainee learning to apply these codes (e.g., J. M. Ray \& Ray, 2008). However, not all continuous observational procedures involve TTC's explicit coding using an exhaustive set of behavioral categories, thus resulting in continuous observation, but without a continuous record of all behaviors occurring during the entire time period. For example, a recent survey by Mudford, Taylor, and Martin (2009) of articles published in the Journal of

\footnotetext{
${ }^{1}$ After the 1957 publication of A Glossary of Terms, Verplanck published an Internet "Preface" statement on the approach taken (http://web.utk.edu/ wverplan/gt57/preface.html), and he spent the remainder of his life extending this early operations analysis approach for defining procedural, empirical, and theoretical terms in the clearest way possible. The greatly expanded result of his pursuit of the development of a common language for discussing behavior is available as an online Glossary and Thesaurus of Behavioral Terms at http://www.ai2inc.com/Products/GT.html.

${ }^{2}$ The Train-to-Code software system is copyrighted and commercially sold and distributed by (AI) ${ }^{2}$, Inc. (www.ai2inc.com). Authors R. D. Ray and J. M. Ray are each stockholders in $(\mathrm{AI})^{2}$, Inc.
}

Applied Behavior Analysis (JABA) from 1995 through 2005 shows a divergent trend that starts with roughly equivalent frequencies of use for continuous and discontinuous recording but subsequently favors the more frequent use of continuous observation methods (but not continuous coding - a distinction we will subsequently elaborate upon) over discontinuous methods. This trend in increasing use of continuous observation correlates quite positively with the increasing availability of miniaturized technologies for data collection in real time over this same period.

We conducted a broader survey of the literature in preparation for having the TTC system ultimately be capable of training observers to carry out any observational procedure used in either research or applied settings. In that broad survey, we focused on empirical features that distinguish each alternative behavioral coding procedure and, subsequently, generated an organizational scheme for systematically classifying, comparing, and contrasting those procedures. In that broad survey and in a subsequent targeted literature survey, we found that discontinuous methods have been the more commonly reported coding procedures. We also sought to specify each of these reported procedures with sufficient precision to guide user-interface development in TTC. The result is a taxonomy suitable for teaching aspiring scientists about alternative observational methods and their implications. In fact, while modeling the second incorporated procedure in TTC (Behavioral Frequency Count), utilizing the procedural specifications in the present taxonomy, we learned so much that we are convinced that even experienced researchers will find issues to further consider within this review.

\section{Classifying observation research procedures}

Few articles have attempted to standardize names for the relevant methodological variations found in the literature on direct observation of behavior. Some articles have addressed specific topics, such as sampling strategies for behavior or individuals (e.g., Altmann, 1974; Friman, 2009) or alternative time sampling procedures and how they compare with actual continuous recording (Mann, Have, Plunkett, \& Meisels, 1991). Some secondary sources (e.g., Bakeman \& Gottman, 1997; Martin \& Bateson, 1993; Sharpe \& Koperwas, 2003; Wallace \& Ross, 2006) have offered comprehensive overviews of procedural alternatives. However, as we attempted to perform an operations analysis of these procedures, we found that descriptions typically lacked sufficient clarity to replicate many of the procedures. Furthermore, authors often conflated factors that should, instead, be treated separately.

In conducting our operations analysis, we surveyed both primary and secondary sources, focusing on disciplines that 
utilize observational approaches extensively, such as applied behavior analysis, child development, and animal behavior. The present article presents an annotated and illustrated classification taxonomy that summarizes procedures found by our search. Although most of the studies used involved visual observations, we did not eliminate auditory or textual observation research. We also attempt to identify procedural variations for which we have yet to find exemplars.

However, before focusing on this taxonomy, four overarching issues need consideration: (1) structural versus functional descriptions of behavior; (2) event versus state perspectives on behavior; (3) mutually exclusive and exhaustive behavioral categories, along with the relevance of R. D. Ray and Delprato's (1989) concept of measurement domains as a classificatory means for parsing concurrently occurring behavioral events and their recording; and (4) methods of calculating IOA. Each issue represents a decision that is best made prior to selecting a procedure from our taxonomy.

Structural versus functional descriptions of behavior

R. D. Ray and Delprato (1989) have offered a detailed account of differences between relying upon structural criteria (topographical or kinesiological) versus functional criteria (consequential or effect defined) for defining behavioral categories. A rather detailed review of the alternative measurement criteria for behavior is available in Friman's (2009) chapter on behavioral assessment. Friman distinguished between primary (direct observation) measures, secondary (indirect) measures, and use of the products of behavior for assessing behavior. Bakeman and Gottman (1997) made a similar distinction in their discussion of a continuum of criteria that range from physical (e.g., postural, physiological measures), on one end, to socially defined codings (e.g., aggressive, playful), on the other end. R. D. Ray and Delprato also discussed some of the problems that may arise when these two alternative approaches are conflated, or mixed into one intended taxonomy (see also Purton, 1978).

Clear examples of physically/structurally defined behaviors are gaits of movement or locomotion (see Collins \& Stewart, 1993). Categories differentiating gaits might include mutually exclusive categories, such as standing, walking, trotting, cantering, galloping, and so forth. Some gaits are so physically based that they may be analyzed and even computer modeled on the basis of physical variations within a category across individuals (see Cunado, Nixon, \& Carter, 2003). Categories describing variations in movement-based gaits may be easily mixed with other nonmovement structural categories to define a singular behavioral taxonomy, such as lying down, sitting, rolling over, or jumping, but typically should not be conflated with more functionally or socially defined behaviors, such as playing or flirting. Playing and flirting are, in fact, apt examples of functional/socially defined categories that reflect the reactions of the observer.

Failing to distinguish between structure and function can lead to confusion as to when a correct coding can be made. Some procedures do not require timing accuracy, while others do. Structurally defined behavior categories can typically be coded very quickly after initiation of a behavior. For example, a person does not have to remain standing very long after transitioning from a sitting position before an observer can reliably identify that standing is occurring. Early identification of a behavior category might be desirable when recording it, since early identification avoids confusing what just happened with what is happening now in the record.

Functionally defined behavior categories, however, often require a judgment based on a completed behavioral episode and cannot be designated near the beginning of an episode (e.g., judging whether the person is making a pun or whether a person is asking a rhetorical question). In such situations, it may be difficult to determine whether the code applies to the recently ended behavior or to the behavior currently in progress. If we allow for some postbehavior or coding tolerance time window within which the previous behavior must be coded, this time window helps to resolve this confusion despite the fact that a new behavior may be in progress. As we will see presently, this resolution is critical for assessing observational accuracy or reliability.

\section{Methodological implications of events versus states}

We often describe a behavior as though it did not extend in time but, rather, occurred at a moment in time (e.g., turned on the light, fired the gun, or closed the door). That is, we are describing this behavior as an event. We locate such events at a particular moment in time, rather than giving them temporal duration. Since behavior is actually a continuous process, these descriptions, of course, represent a convenient fiction. All behavior is extended in time. At the other extreme, we describe some behavior as a process spread out in time (e.g., mood, attitude, personality). That is, we describe this behavior as a state. We may try to digitize the flow of such behavior across time by locating the time when the state begins (e.g., falling asleep), but we consider that this behavior perdures - at least until some ending time (e.g., waking).

There is, of course, a continuum between describing behavior as event and describing behavior as state, and these actually represent potentially helpful perspectives, rather than facts about behavior. We expect personality to perdure and are surprised when people change (assumed: 
their behavior). We expect a meal to be a temporary state. We often extend a series of events to emphasize that they represent a bout of behavior (i.e., a temporary state). In this report, we merely mean to acknowledge this continuum, not to judge the value of event versus state descriptions. For some purposes, one will be best; for other purposes, the other. Yet there are methodological implications. If the observer chooses to record the frequency of the behavior, that emphasizes behavior as an event. If the observer chooses to record the duration of the behavior, that emphasizes behavior as a state. A continuous record of behavior is neutral with respect to event versus state and can be used to focus on either frequency or duration. However, one needs to avoid conflations of event-based categories with state-based categories, because they typically cannot be compared.

Another issue of comparison legitimacy arises even if a researcher chooses to define all behaviors as state phenomena. If categories with radically different state durations, such as sneezing and sleeping, are incorporated into the same coding scheme, comparing such states, on the basis of either frequency or duration, can be highly problematic. R. D. Ray and Delprato (1989) have emphasized micro- vs. macro-event resolution as a meaningful way of dealing with categorical disparities in such parametrics, and coding schemes will avoid numerous problems by adhering to some standard of relative homogeneity in their level of resolution for categories. Other recording strategies described below will emphasize one perspective or the other (i.e., event vs. state; micro vs. macro). Often the choice of procedural parameters (e.g., length of observing period, length of time sample within the observing period) will align well or badly with the chosen perspective.

Methodological implications of mutually exclusive and exhaustive taxonomies of behavior

Designing a behavioral taxonomy that provides the information you desire in the most efficient manner is a challenging task. Adding more categories will provide a more complete picture; reducing the number of categories may well reduce error. What is the right balance? Video and/or computer-aided observation often improves observer accuracy and precision, and thus their use will allow us to increase the number of acceptable categories. But is the added information overkill? Allowing for a process of trial and improvement as you design a taxonomy for your observation project is good advice. Two issues to consider in this development are the degree to which your behavioral categories are mutually exclusive and to which they are exhaustive.

Two categories are mutually exclusive if the observed individual can be doing one (e.g., walk) or the other (e.g., run), but not both, at a given time. The example, however, (walk vs. run) was chosen to indicate that work will be required to develop a definition to clearly distinguish one from the other, that the definition should be one that others will understand, and that the definition will have important implications for what your observations will tell you. Should there be an intermediate category (e.g., jog)? Should a more extreme category be designated (e.g., sprint)?

Among the issues to be addressed in developing a behavioral taxonomy is what to do with categories of behavior that are not mutually exclusive (e.g., walk, chew gum). R. D. Ray and Delprato (1989) introduced the concept of descriptive measurement domains to separate categories into mutually exclusive sets. Any two categories that are not mutually exclusive may be placed into separate domains. Ideally, a domain involves a consistent focal point, such as postural versus oral activities in the walk/ chew example above. Alternatively, one domain may focus on postural configurations, such as standing, walking, sitting, and so forth, while another focuses on verbalsocial interaction events initiated by the same individual, such as instructing, flirting, kidding, threatening, and so forth.

We can, of course, always create combination categories and, thereby, force our independent categories to be mutually exclusive-for example, walk while chewing, walk while not chewing, not walk while chewing, not walk while not chewing. We have thereby created four mutually exclusive categories by combining the two independent categories. In the analysis phase, any multidomain comparison accomplishes exactly this kind of combinatorial description post hoc from concurrent or synchronized single-domain recordings (see Astley et al., 1991). The observer's task, however, has been made more difficult by requiring both a behavior category and a domain to be observed and recorded simultaneously, and thus, the coding has been made less reliable.

A related issue is the extent to which the behavioral categories being used are exhaustive - that is, whether they will allow each and every moment in the behavior stream to be categorized. This issue arises only under conditions where a continuous record of all behaviors is the goal. For example, if our categories are stand versus walk, what code would we use for sit? There is always a default approach that makes your list exhaustive by adding a residual category, such as other. But such a category is defined only by exclusion and may well be a category that observers find difficult to use (see Wallace \& Ross, 2006, for a far more elaborate discussion of this issue on the basis of what they call the "bucket" category). Nonexhaustive coding schemes decrease the difficulty of observation but disallow some analyses, as will be detailed below. 
Issues attending interobserver agreement

Suen and Ary (1989) have presented a compelling case for applying much of psychometric theory, including reliability and validity, to observational data and analyses. On the other hand, Lee and Del Fabbro (2002), as well as Wallace and Ross (2006), have argued for a Bayesian approach and have detailed various concerns with the frequentist approach underlying psychometric strategies. Perhaps the most commonly used concepts underlying observational research are those of agreement, accuracy, and reliability. Suen and Ary argued that theory of measurement is highly germane to understanding these issues. They reviewed mutually contradictory definitions for agreement versus reliability versus accuracy commonly found in the literature. For example, Bakeman and Gottman (1987) treated agreement as two observers agreeing between themselves and used reliability as a measure of how true a measure is. Alternatively, Martin and Bateson (1993) used reliability as an absence of random errors and used accuracy as an absence of systematic errors. Hartmann and Wood (1992) based agreement on the degree to which raw scores match and reliability on the degree to which standard scores match. Their preferred measure was accuracy, which they asserted is a match between observer and an external criterion. Finally, Cone (1982) aligned the term accuracy with validity but found differences between all three concepts of reliability, accuracy, and validity. Suen and Ary pointed out that most of these positions are mutually contradictory and, thus, reflect differences in theoretical origins.

It is far from our present purpose to offer a full discussion of, or resolution for, such discrepancies and differences of opinion. Neither is it our purpose to detail the many implied measurement tactics or their associated degrees of statistical confidence. These issues are far too complex to deal with here. Beyond that, new approaches and solutions continue to evolve. What the present section does present is a simple classification of commonly used strategies and tactics, along with respective references to appropriate sources for accessing elaborations of each. Our presentation of these issues is also an affirmation of Vollmer, Sloman, and St. Peter Pipkin's (2008) assertion that, especially in the monitoring of treatment integrity, there are critical and practical dimensions that make the use of some measure of IOA and/or data reliability truly imperative.

We will begin our overall referenced classification by adopting one convenient distinction offered by Suen and Ary (1989). They used IOA to label processes and indices for measuring the degree to which two or more observers agree with one another. They also suggested intraobserver reliability (IOR) as a term for estimating the consistency with which a single observer might record the same behavior in multiple instances. They offered arguments for markedly different measurement tactics for these two different issues and suggested that IOA is the less complicated reliability issue. Many, if not most, authors do not seem to agree, given their common application of the same indices for both IOA and IOR. The issue is sufficiently complex that Suen and Ary offered a substantial portion of one chapter detailing what they considered to be uniquely applicable techniques for assessing IOR.

IOA measures typically begin with code-matching techniques between observers. This can be as simple as Suen and Ary's (1989) smaller/larger index for comparing two frequency counts of the same behavioral category or may encompass a more broadly applicable percentage of agreement (Mitchell, 1979; Suen \& Ary, 1989), or what is sometimes called simple percent agreement (Bakeman \& Gottman, 1997). Despite its common and continuing use (Mitchell, 1979), percent agreement has many critics (Bakeman \& Gottman, 1997; Hartmann, 1977; Hartmann \& Wood, 1992; Mitchell, 1979; Suen \& Lee, 1985). On the other hand, it also has its defenders (Baer, 1977; House, Farber, \& Nier, 1983). One commonly cited weakness is its failure to discount potential agreements that may result from chance (Bakeman \& Gottman, 1997). The most common solution offered for this shortcoming is the use of Cohen's (1960) kappa coefficient (see also Bakeman \& Gottman, 1997; Mitchell, 1979; Suen \& Ary, 1989), although an approach based on occurrence and nonoccurrence agreement indices has also been used to adjust for chance agreements (Suen \& Ary, 1989). There are also substantial arguments against using kappa because of its sensitivity to unequal base rates of various categories (Grove, Andreasen, McDonald-Scott, Keller, \& Shapiro, 1981; Guggenmoos-Holzmann, 1993; Spitznagel \& Helzer, 1985).

The many problems associated with code-by-code comparisons have led some authors to apply more classical psychometric approaches to measuring IOA. For example Mitchell (1979) and Suen and Ary (1989) each reviewed the typical employment of various forms of correlation techniques, ranging from simple Pearson $r$ correlation applied to two observers to intraclass correlations, including split-half or alternate-form and even test-retest assessment techniques. Wallace and Ross (2006) argued that correlations are too generalized and suggested, instead, a category-by-category analysis based on signal detection concepts of sensitivity versus specificity, as commonly used to describe receiver operating characteristic curves.

However, there is yet a third approach, which many assert is perhaps the best of all options - largely, because it is so robust. It is called generalizability theory (Cronbach, Gleser, Nanda, \& Rajaratnam, 1972) and has been reviewed by Campbell (1976), Mitchell (1979), and Suen and Ary 
(1989). Bakeman and Gottman (1997) have called it a conceptual breakthrough, and Mitchell (1979) has suggested that the generalizability approach should be used to identify error sources in addition to those involving the observer. As Mitchell pointed out, Cronbach's approach is somewhat analogous to factorial analyses in experimental research, in that the analysis allows one to compute variance component contributions from multiple facets of the project. Thus, one might use generalizability coefficients to reflect variance contributions from subjects, categories, scorers, and even their interactions (Mitchell, 1979). This flexibility makes generalizability an appropriate means for assessing either IOA or IOR (Suen \& Ary, 1989). Unfortunately, despite its power and appeal, it is beyond our present scope and purpose to detail the complexities of the generalizability approach, and interested readers should consider the reviews cited above for elaborations.

Hartmann (1977) has distinguished between agreement issues that involve concerns with consistencies in observation intervals, stability of IOA over time (i.e., observer drift), and stability across various situations and/or behavioral categories, as well as reliability of the basic data themselves. Generalizability theory allows for the possibility of incorporating each of these issues as facets of a single analysis if one's research is designed to systematically account for each.

Beyond the alternatives

For our present purposes, the critical elements in all of these alternatives is how the procedural taxonomy we are about to detail impacts one's options for applying any given technique, once one has decided which technique is most suited to the purpose of the research. In many cases, the observational procedure will preclude some analyses while supporting others, as we will discuss later. Most germane to the present discussion is the point made by Friman (2009) that an adequate observation procedure should allow for adequate assessment of at least some measure of IOA or IOR. This assertion implies that the selected procedure must provide information necessary for that assessment. If all behavioral codes and their location in time are recorded (i.e., continuous coding), virtually any or all types of agreement calculation are possible. But most research is not able to accommodate this comprehensive procedure.

Consider a case where two observers record the following sequences (see Table 1) and we are to judge their agreement. In the table, observer 1 records $A, A, B, A$, $\mathrm{C}, \mathrm{B}, \mathrm{A}, \mathrm{B}, \mathrm{C}, \mathrm{A}$, and observer 2 records $\mathrm{A}, \mathrm{A}, \mathrm{A}, \mathrm{B}, \mathrm{C}, \mathrm{B}$, $\mathrm{B}, \mathrm{B}, \mathrm{C}, \mathrm{A}$. Without recording time, it will be difficult to resolve apparent discrepancies in alignment. Thus for entry 3 in Table 1, where observer 1 records $B$ and observer 2 records $\mathrm{A}$, did observer 1 miss an observation (i.e., the third A recorded by observer 2)? And if the event was missed,
Table 1 Hypothetical coding of the same behavioral events by two independent observers

\begin{tabular}{ll}
\hline Behavioral Record & \\
\hline Observer 1 & Observer 2 \\
\hline A & A \\
A & A \\
B & A \\
A & B \\
C & C \\
B & B \\
A & B \\
B & B \\
C & C \\
A & A \\
\hline
\end{tabular}

how does such an omission error enter into the analysis, since no category is recorded. Alternatively, did observer 2 record a single event twice (thus counting an extra A event)? And if so, how is that coding handled, since it has nothing to be compared against? And if either of these are the case, how do we realign our remaining observations for comparison? If time is recorded, realignment may be easier to accomplish but will not resolve the code-matching problem. Thus, one must consider how contiguous in time and/or sequence one's observations must be to count them as being in agreement.

The last issue is rarely described in sufficient detail within research reports for readers to truly understand how researchers actually matched codings for the IOAs they report. Neither are most methodological textbooks or articles sufficiently clear in their procedural guidance to allow researchers to anticipate and solve these issues. Consider the following case-in-point, for which we have found only one reference (i.e., Bakeman \& Gottman, 1997) that addresses the topic sufficiently to offer any understanding of the issues involved. Assume that a continuous observation procedure, soon to be detailed, is being used and that this procedure relies upon a mutually exclusive and exhaustive set of categories. Such a procedure would have each observer record both the category currently in progress and the start and stop times of that category. This of course preserves all possible information, including sequence and temporal contiguity, that defines the stop time for one category, as well as the start time for the next.

But what if such a procedure is using video recordings for the coding of a given target participant and the observer records start/stop times within the accuracy allowed by the frame rate of the video? Typically formatted NTSC video records/plays at approximately 30 frames per second (fps). Using frame-by-frame analysis, do we expect the agreement to be exact to the actual frame (i.e., 1/30th of a second)? If not, what is our tolerance window that allows for us to 
discount or ignore differences in frame specifications between two independent observer recordings? Bessel's famous personal equation defines individual differences as being unavoidable in detecting transitional events (Sanford, 1888). If we cannot expect an exact frame match for when each behavioral transition occurs, what might be a reasonable tolerance window in which any mismatch between observer A and observer B will be counted as a match?

To clarify just one of the implications of alternative answers to this question, consider Figs. $1 \mathrm{a}, \mathrm{b}$ as alternative examples of continuous coding. These examples illustrate an event-defined (1a) versus state-defined (1b) approach to IOA measurement. The upper blue and aqua bars represent two different, temporally sequential actual behaviors being coded. The second bar is an observer's recording of these same events. Using a simple count of each behavioral event for measuring coding accuracy, and using actual versus coding as our determination of agreement $(+)$ or disagreement (-), as illustrated in Fig. 1a, we would code/count as follows: blue versus blue $(+)$, blue versus aqua $(-)$, and aqua versus aqua $(+)$. Using a simple percent agreement, we have 2 agreements divided by 2 agreements plus 1 disagreement, or 3 total codings, for a ratio of $2 / 3$, or $67 \%$ agreement on the behavioral counts being made.

But what if we wished to focus on the amount of time in agreement, as illustrated in Fig. 1b? Here, the actual agreement in behavioral states accounts for 14 of 17 total time units (30th of a second). That calculation changes the agreement to $82 \%$ of the time. This major difference in percent agreement derives merely from a shift in comparison perspective. Which is correct? Furthermore, while we do not illustrate it in the example, this change in perspective

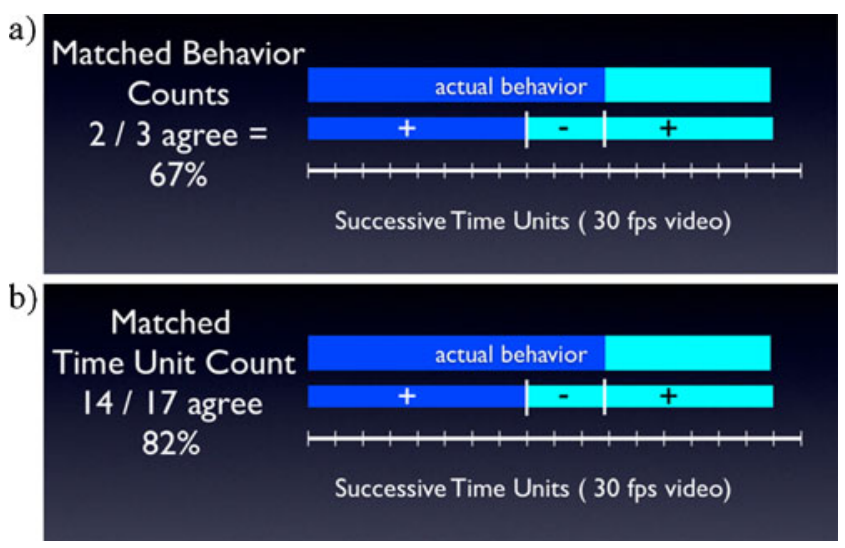

Fig. 1 Two approaches to calculating agreement. Percentage of agreement between the actual occurrence of behavior and an observer's record of that behavior may be calculated using a count of the number of instances of agreement and disagreement (a, showing $67 \%$ agreement) or b a measure of the amount of time when the observer's record and the actual behavior were in agreement (b, showing $82 \%$ of the $30 \mathrm{fps}$ video frames as time in agreement) impacts Cohen's kappa through kappa's correction for chance agreements (i.e., the subtracted chance, or $p(\exp )$, factor used in kappa calculations is .44 in the case of event count comparisons versus .49 in the case of time-inagreement comparisons). Thus, perspective (event vs. time) matters.

So which of these IOA calculation strategies should be used when a continuous coding procedure is being followed and two observer records are to be compared? And if we follow one rule in one procedure (e.g., simple frequency count) and another rule in an alternative procedure (e.g., continuous coding), what comparisons may be made between the procedural impacts on IOA? Unfortunately, while it may be relatively easy to discern whether behavioral categories being used in any given research publication are treated as states or entities, it is nearly impossible to determine from the method sections of most publications whether behavioral counts or synchrony in total behavioral states are the basis for the reported IOA calculations!

We became acutely aware of the event-agreement versus time-in-agreement differences when programming these metrics for TTC. It is worth noting that in most situations where one is training new observers, it is common to consider the developer who established a given taxonomy or coding scheme as the reference coder against which trainees are compared for IOA. In such circumstances, the developer is considered to be an expert on the application of the taxonomy, and thus it is common to take their coding as "correct" and to define the trainee's agreement as accuracy (see Boykin \& Nelson, 1981). Thus, we followed that tradition in TTC. This does not assert that the developer has established a gold standard for comparisons, but only that, in training, we must use some reference criterion. And in doing so, we deem coding accuracy the appropriate term to use for trainee agreement with an expert.

Interestingly, a study was published by Mudford, Martin, Hui, and Taylor (2009) well after our current TTC design decisions had been made and our considerations of time in agreement versus event agreements had been applied. Mudford and colleagues' study is one of the rare published comparisons of algorithmic impact on IOA, as well as agreements, of which we are aware. The authors compared 12 observers recording the same behavioral records using hand-held computer recorders. Low, medium, and high rate occurrences of both discrete-event and state-durational measures were compared for IOA, as calculated between observers, as well as for accuracy - that is, comparing against a criterion record. Three agreement/accuracy algorithms were compared, including exact agreement, blockby-block agreement, and time window analysis. Also, they studied the relative impact of increasing tolerance windows, 
ranging from zero to $\pm 5 \mathrm{~s}$. Details of their findings are complex, but it suffices for our purpose to note that virtually all variables they investigated - type of behavioral definition, rates/durations, and algorithm used-had differential impacts on agreement/accuracy values!

Perhaps the conclusion to draw for our present discussion is that an observation procedure should provide sufficient information to allow a convincing IOA to be performed according to extant standards. Unfortunately, sufficient information is not always supplied. For example, in their rather extensive analysis of interobserver agreement calculation methods reported in 10 years of articles published in JABA, Mudford, Taylor, and Martin (2009) reported that there were sufficient differences in published descriptions of interobserver agreement algorithms as to require clarification correspondence with many senior authors. It is important that sufficient detail be provided in publications to allow readers to determine their level of trust in the observations and to more accurately replicate the reported procedure. But that information clearly requires that researchers clarify the behavioral unit versus temporal unit foundations of their measurements. It is important that researchers be aware that standards and expectations change, both from journal to journal and across time. Thus, the present taxonomy, to which we now return, will attempt to identify not only observation and recording procedures, but the attendant IOA/IOR analysis potentials as well.

\section{A taxonomy of procedures used for behavioral observation}

Having reviewed significant issues that must be addressed regardless of the procedure used for observation, we remind the reader that we developed the present procedural taxonomy by selectively searching for exemplars for operations analysis. We actually conducted two literature reviews, with the first being more expansive and focused exclusively on reported methods where the only criterion for our retention was the reported use of a direct observational procedure that added to our growing collection of such procedures. It was this collection that was operationally analyzed to build our procedural taxonomy (actual references eventually used for examples of each distinct procedural operation are included in the Supplemental Materials for this article). The second literature review focused on a selected volume of two representative but disparate journals, to determine which, if any, of our predefined procedures were used within publications contained in that volume. These outcomes are reviewed in a subsequent section of this article (see "Is the Taxonomy Exhaustive"). As was noted, for our first review, we looked for as many variations of systematic coding procedures as we could find and inductively derived the two common dimensions (i.e., behavior and time) from all exemplars found. We then proceeded to define a taxonomy on the basis of intersections of the distinct subclassifications of those dimensions that emerged (i.e., cells in a procedural matrix). Behavior and time are therefore the two defining dimensions of the taxonomy illustrated in Table 2. It is worth noting that this taxonomy allows us to describe all studies using direct observation of behavior that we encountered in our literature search. Below, we will describe each of these procedures and will offer one or more exemplary studies for those we could document. Some cells reflect a use of behavior and time in a manner that is not a logically feasible procedure. These cells are marked with a "-" symbol in Table 2. Other cells, marked with a "+" symbol, reflect a use of behavior and time in a manner that is logically possible, but no exemplar studies were found in our operations analysis literature search.

Table 2 may be used as a specifications guide for programmers developing algorithms and interface design for computer-assisted data gathering like those we designed into TTC As such, each cell in Table 2 may be expanded in detail (see Supplemental Materials, Tables 116) to become a user's help system that clarifies for research administrators and/or trainees how each selected procedure works if implemented, or would work if not, within such a system. Thus, specifics of how time and behavior are used to define each procedure represented by the cells in Table 2, along with acceptable behavior assessment measures, IOA implications, and cited research examples that illustrate research based on each procedure are included in our Supplemental Materials tables (SMTs). Each SMT will be referenced where appropriate for readers interested in these corresponding operations analysis details and examples.

To also illustrate the subtle but important issues that arise in computer interface design, the two concrete in situ examples currently implemented within TTC are presented in a subsequent section. These examples are included because they give concrete illustrations of functional data collection algorithms and interface design differences and implications. Such a reference to software development emphasizes the requirement, imposed by all programming tasks, that one develop a complete verbal description of each procedure implemented prior to creating a software model of that procedure. Both interface and algorithmic expression provide important evaluative feedback on the precision and completeness of one's procedural descriptions. It was this requirement that guided us in assessing the completeness of our operations analysis of the literature and our development of the present taxonomy. 
Table 2 Types of behavior and time recording that define various types of behavior observation procedures

\begin{tabular}{|c|c|c|c|c|c|}
\hline \multirow[b]{2}{*}{ Types of Time Recording } & \multicolumn{5}{|c|}{ Types of Behavior Recording } \\
\hline & Yes/No & Dominant & Whole-Interval & Frequency or Duration & Sequence \\
\hline During the observing session & $\begin{array}{l}\text { 1. Session: Yes/no } \\
\text { occurrence }\end{array}$ & $\begin{array}{l}\text { 2. Session: Dominant } \\
\text { behavior }\end{array}$ & + & $\begin{array}{l}\text { 3. Session: Behavior } \\
\text { Frequency/Duration }\end{array}$ & $\begin{array}{l}\text { 4. Session: Behavior } \\
\text { Sequence }\end{array}$ \\
\hline $\begin{array}{l}\text { At the start } \text { or } \text { end } \text { of each } \\
\text { observing interval/trial } \\
\text { within the observing } \\
\text { session }\end{array}$ & $\begin{array}{l}\text { 5. Momentary time } \\
\text { sampling: } \mathrm{Y} / \mathrm{N}\end{array}$ & + & - & $\begin{array}{l}\text { 6. Momentary Time } \\
\text { Sampling: Frequency }\end{array}$ & - \\
\hline $\begin{array}{l}\text { Within each observing } \\
\text { interval/trial within the } \\
\text { observing session }\end{array}$ & $\begin{array}{l}\text { 7. Complete interval } \\
\text { time sampling: Y/ } \\
\mathrm{N} \text { (often called } \\
\text { partial interval } \\
\text { sampling) }\end{array}$ & $\begin{array}{l}\text { 8. Complete interval time } \\
\text { sampling: Dominant } \\
\text { behavior }\end{array}$ & $\begin{array}{l}\text { 9. Complete interval time } \\
\text { sampling: Whole- } \\
\text { interval behavior }\end{array}$ & $\begin{array}{l}\text { 10. Complete interval time } \\
\text { sampling: Frequency/ } \\
\text { duration }\end{array}$ & + \\
\hline $\begin{array}{l}\text { Within each observing } \\
\text { interval/trial and recorded } \\
\text { during the following } \\
\text { recording interval }\end{array}$ & + & $\begin{array}{l}\text { 11. Alternating interval } \\
\text { time sampling: } \\
\text { Dominant behavior }\end{array}$ & + & $\begin{array}{l}\text { 12. Alternating interval time } \\
\text { sampling: Frequency }\end{array}$ & $\begin{array}{l}\text { 13. Alternating interval time } \\
\text { sampling: Sequence }\end{array}$ \\
\hline $\begin{array}{l}\text { Time stamp recording of } \\
\text { events within the observing } \\
\text { session }\end{array}$ & $\begin{array}{l}\text { 14. Event time stamp } \\
\text { recording: Time of } \\
\text { momentary event } \\
\text { occurrence }\end{array}$ & - & - & $\begin{array}{l}\text { 15. Partial continuous coding: } \\
\text { Start and end times for } \\
\text { nonexhaustive behavioral } \\
\text { events }\end{array}$ & $\begin{array}{l}\text { 16. Continuous coding: start } \\
\text { times for exhaustive } \\
\text { behavioral events (end } \\
\text { times derived) }\end{array}$ \\
\hline
\end{tabular}

Some cells reflect a use of behavior and time in a manner that is not a logically feasible procedure. These cells are marked with a "-” symbol in Table 2. Other cells, marked with a "+" symbol, reflect a use of behavior and time in a manner that $i s$ logically possible, but no exemplar studies were found in our literature search.

\section{Approaches to behavioral recording}

Each time a behavioral observation is recorded, a type of behavioral measurement and a type of time measurement is required. There are several alternative types of behavioral measurement, as illustrated by the columns of Table 2 . The first type is a simple dichotomous judgment resulting in a one/zero or yes/no entry to indicate whether the behavior did or did not occur or to make some social/functional judgment of its occurrence (e.g., playing, fighting, or correct/incorrect responding). The second type records only the dominant behavior that occurred within the complete observation period (i.e., the behavior that occupied the greatest portion of the observation period). The third type involves recording any behavior that occurred throughout the complete observation period. The fourth type designates the total number or total duration for behavioral events of each type that occur within a complete observation period or the latencies until a behavior occurs following some environmental event. In the fifth type, the sequence of behaviors as they occur during a complete observation period is recorded.

Observational records must also use one of three alternative approaches to incorporating time into the record. Observers may record only the number of behavioral events (or the duration of these events) within the total observation period (Row 1 of Table 2), may segment the observation period and make a separate recording for each segment (Rows 2, 3, and 4 of Table 2), or may use continuous recording, noting the time of each behavioral event within the overall observation period (Row 5 of Table 2). We thus elaborate the options for using these types of time measurement and their impact on the behavioral observation record.

Time of occurrence is ignored in the record In the first option, time is not recorded but is used only to define the beginning and end of the total observation period. This is the simplest use of time, since it references only the session for observing and does so only by the frequency and duration of sessions (e.g., four 20-min sessions) or the start/ end time of each. Within the family of procedures using only such a time reference are those that seek to record whether or not one or more behaviors of interest occurred at all during the session (see Fig. 2, Procedure 1, with more precisely defined operations and examples in SMT 1, Session: Yes/No Occurrence), which behavior dominated the session, relative to all alternatives (see Fig. 2, Procedure 2 and expansions in SMT 2, Session: Dominant), the frequency or total time spent in one or more behaviors of interest during the session (see Fig. 2, Procedure 3 and expansions in SMT 3, Session: Behavior Frequency/ Duration), or the exact sequence of behavior(s) during a given session (see Fig. 2, Procedure 4 and expansions in SMT 4, Session: Behavior Sequence). While it may be logically possible to record behaviors that fill the entire observation period (e.g., slept throughout the night), we did not find any examples using such a procedure and, thus, have not enumerated it.

Segmenting observation periods by time intervals or trials Each observation period may be divided into equal 


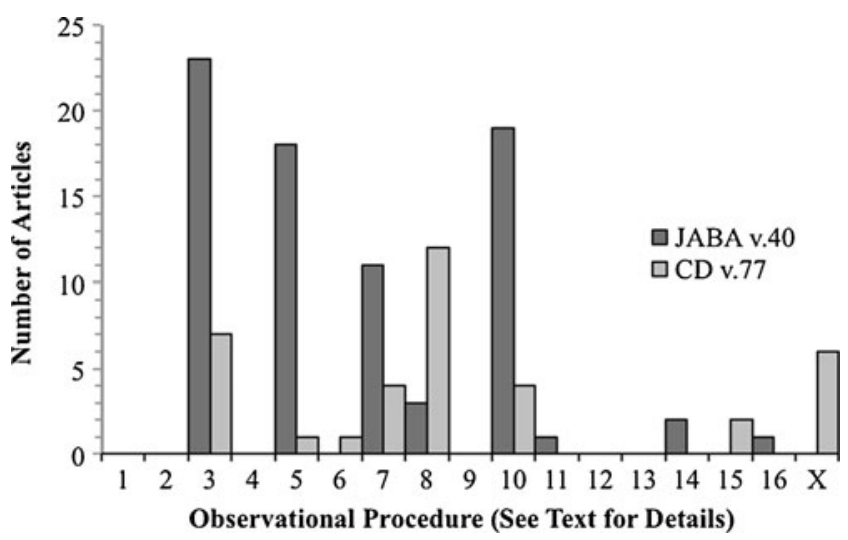

Fig. 2 Distribution of use of each type of direct observational procedures published in a single volume of two research journals. All research reports in one volume of the Journal of Applied Behavior Analysis (Vol. 40, 2007) and of Child Development (Vol. 77, 2006) were reviewed. The ordinate shows the number of studies found within these research articles that used each of the 16 procedures outlined in Table 2. Categorization of procedure was carried out by the authors, and agreement was achieved through a procedures-operations analysis of stated methods and discussion. The number of procedures used is greater than the number of articles in the volumes that used behavioral observation, since a single research report often contained multiple studies. The number of studies utilizing behavioral observation procedures that could not be categorized as one of the 16 procedures in Table 2 is shown in the rightmost column. Those six studies whose methods could not be categorized reported example phrases from field notes or post hoc content analysis of field notes and, thus, were considered not to be using true coding procedures. All studies that actually used systematic coding of observations could be categorized

time intervals (interval recording) or into separate trials (trial-by-trial recording). By creating a separate record for each interval or trial within the overall observation period, changes in behavior can be tracked across that period. A description for each approach may be helpful.

For interval recording, a signal marks a time for the observer to observe, record, or both. Thus, behavior may be observed at some marker separating intervals (momentary time sampling; Row 2 of Table 2) or may be observed throughout each interval and recorded at the end (complete interval time sampling; Row 3 of Table 2), or the intervals may be further divided into alternating observation and recording subintervals (alternating interval time sampling, also known as intermittent interval time sampling; Row 4 of Table 2).

For trial-by-trial recording, a trial consists of a cycle of events defined by environmental conditions and the participant's behavior. For example, in a particular experiment, an intertrial interval is followed by presenting a question to the participant, who, in turn, produces or selects his or her answer to end the trial and, thereby, starts the next intertrial interval. As for interval recording, in trial-by-trial procedures, behavior may be observed at the end of the trial or throughout the trial, or subintervals may be specified for observing within the trial. There seems to be no common vocabulary for labeling these distinctions.

The next three rows in Table 2 share the common feature of dividing the observation period into intervals or trials for successive recordings. What distinguishes each of these rows is whether observations are made of the behavior occurring at the beginning/end of each interval or during each interval and, if the latter, whether the observation interval is concurrent with recording or offers a separate interval for recording. In Row 2, the family of procedures includes only two found examples.

The first (see Fig. 2, Procedure 5 and expansions in SMT 5, Momentary Time Sampling: Y/N) focuses upon recording only whether or not behaviors of interest are occurring at the interval reference (start or end), while the second (see Fig. 2, Procedure 6 and expansions in SMT 6, Momentary Time Sampling: Frequency) records the number of instances, items, or individuals that meet the observation criterion at the interval reference.

Of the interval options, only complete interval time sampling (see Table 2, Row 3) allows for a full accounting of all the time within the overall observation period. This approach provides records of frequency, durations, or cumulative durations within each interval. Thus, Row 3 depicts a family of procedures that share a use of intervals of time to prompt recording, but behaviors that are recorded occur at any time within the interval, and not necessarily only at the termination, as do those in Row 2. These procedures may differ only in whether they involve recording a yes/no occurrence for behaviors at any time in the interval (see Fig. 2, Procedure 7 and expansions in SMT 7, Complete Interval Time Sampling: Y/N), recording the behavior that meets criteria for dominance in the interval (see Fig. 2, Procedure 8 and expansions in SMT 8, Complete Interval Time Sampling: Dominant Behavior), recording behavior that consumed the entire interval (see Fig. 2, Procedure 9 and expansions in SMT 9, Complete Interval Time Sampling: Whole-Interval Behavior), or recording frequencies/durations for any behavior occurring within the interval (see Fig. 2, Procedure 10 and expansions in SMT 10, Complete Interval Time Sampling: Frequency/Duration).

As with other families, there is a logical alternative procedure for which no example was found and, thus, is not enumerated. This would involve recording behavior sequences at the end of each interval but that occurred across each interval (see Table 2, Row 3, Column 5). Technically, this would be very difficult to accomplish, since it implies observing an on-going sequence while recording a previous sequence.

It should be noted that each measure described above has implications for data analysis possibilities. Frequency recording allows analysis of frequency, relative frequency, and rate (frequency/length-of-interval or frequency/dura- 
tion-of-trial). Recording each event's duration by category allows an extrapolation of these same frequency analyses, as well as descriptive statistical summaries (e.g., mean and standard deviations for each behavior's temporal dynamics, including total time spent in each behavioral state). Cumulative durations allow only the latter analysis (i.e., total time spent in each behavioral state). Since each measure accounts for all time in each interval, intervals may be used as an integral part of the analysis of the entire observation period. Thus, complete interval time samplings allow researchers to use each interval or trial as a window for successive time series analyses within a total observation period, as well as allowing an overall descriptive accounting of the total observation period.

Row 4 depicts a family of procedures that share the use of alternating successions of observation interval followed by recording interval. We found this use of time recording to exist for only three types of behavioral recording. One procedure involves recording, during the recording interval, any behavior meeting the criteria for dominance in the observation interval (see Fig. 2, Procedure 11 and expansions in SMT 11, Alternating Interval Time Sampling: Dominant Behavior). The second procedure uses the separate recording interval to record only the behavioral frequencies occurring during the preceding observation interval (see Fig. 2, Procedure 12 and expansions in SMT 12, Alternating Interval Time Sampling: Frequency). The third procedure involves using alternating recording intervals to record the sequence of behaviors occurring during the immediately preceding observation interval (see Fig. 2, Procedure 13 and expansions in SMT 13, Alternating Interval Time Sampling: Sequence). Other alternating interval procedures logically seem feasible (see Table 2, Row 4, Columns 1 and 3), but we found no example publications using either procedure.

All alternating interval procedures yield incomplete accounts and do not allow measurement of true behavioral rates, durations, or accumulated times. However, by taking into account the proportion of the session devoted to observing, one can estimate true rates, durations, and cumulative times. Estimates of relative frequency, relative duration, and relative cumulative duration by behavior are possible even without considering the proportion of the interval devoted to observing. Thus, if your taxonomy includes several behavioral and/or environmental categories and/or if the recording is difficult to complete (e.g., recording the sequence), intermittent interval time sampling makes recordings possible in real-time circumstances and may be preferred despite the shortcomings of imposed estimates. On the other hand, if the recording is quite simple (one/zero, frequency with a small taxonomy, dominant behavior, whole-interval recording), there is less pressure to use intermittent interval time sampling.
For trial-by-trial procedures, behavioral events at the end of the trial or within the trial are used to prompt recording. The same behavioral data described for interval procedures may be created. In trial-by-trial procedures, it is rare to find a recording of either the temporal latency of response or the duration of each trial. Thorndike's $(1898,1899)$ use of escape latency is an exception that exemplifies the technique in use. However, since the duration of trials is typically defined by a behavioral state (e.g., question has been answered), rather than by the passage of time, the ability to determine the true rate of a category of behavior typically is lost in trial-by-trial approaches. When latency or duration is used, it clearly incorporates time as a fundamental dimension of the trial-by-trial procedure. And when records do include durations, overall rate, and perhaps overall duration, measures may be available. Thus, if time and frequency are both recorded for each phase of each trial, rate of behavior may be determined (e.g., as is done in the operant study of chained and concurrent chained schedules of reinforcement; see Kelleher \& Gollub, 1962, or Herrnstein, 1961).

Continuous observations When observing continuously within any given observation session, time can be brought into the recording by time stamping each behavioral occurrence that is observed across the entire observation period (Row 5 of Table 2.) We should emphasize that such time stamping may be done in one of two ways. In the first option, one time marker is made for each recorded event designating the time when a behavioral event occurred (see Fig. 2, Procedure 14 and expansions in SMT 14, Procedure 14-Event Time Stamp Recording). Alternatively, one can record both a start and a stop time for each behavioral state (see Fig. 2, Procedure 15 and expansions in SMT 15, Procedure 15, where the behavioral categories are not considered to be exhaustive), or only the time when a behavioral state started (see Fig. 2, Procedure 16 and expansions in SMT 16, Procedure 16, Continuous Coding) and subsequently use the end of one behavior as also indicating the time when the next behavior starts, in circumstances where the behavioral categories are considered to be exhaustive. Thus, within the alternatives for continuous observation, it is continuous coding that allows the most complete record of any of the procedures, so that frequency, relative frequency, rate, and sequence of behavior are all directly available from the record, as is the time between each occurrence of a given category (interresponse time). If both the start and end of each behavioral event are recorded or derived (Procedures 15 and 16), the duration of each behavior is also available.

It should finally be noted that time stamp recording approaches are relatively challenging to the observer, although video and/or computer-aided observation tools 
often allow observers to meet this challenge. Such aided observation allows for more complex recordings, including, for example, the use of coding schemes with more numerous behavioral categories, multiple behavioral domains, and even the inclusion of environmental conditions - a topic to which we now turn.

Approaches for including the environment as part of the record

Most researchers would agree that knowing the environmental conditions concurrent with behavior provides a context for the description of behavior that is as important as knowing the behavior itself. Thus, the ideal behavioral record would include a concurrent description of the environmental conditions associated with behavior. In social situations, the behavior of others would be treated as an environmental condition. In some cases, the environmental description can be viewed as being static and, thus, described for the whole observation period (i.e., is contextual). In other cases, environmental events must be considered to be dynamically changing along with the behavior (see Astley et al., 1991; Hawkins, Sharpe, \& Ray, 1994). The most complete record would include timestamped environmental, as well as behavioral, events or states. Optionally, if an interval or a trial-by-trial approach is being used, both environmental and behavioral events may be recorded by interval or by trial to locate them within the observation period.

The behavior analysis tradition is one that strongly favors mixing environmental with behavioral descriptions (Skinner, 1938). This approach often takes what is called an $A B C$ approach, with $A$ indicating immediately antecedent environmental conditions, $B$ indicating the behavior being observed, and $C$ indicating conditions that follow and depend on the behavior (i.e., response-contingent or consequent events). All of these three factors are considered to be necessary elements in a behavioral description (called a three term contingency in Holland \& Skinner, 1961). To be complete, then, a continuous behavioral record would need to categorize antecedent conditions, behavioral events or states, and consequent outcomes. And an interval or trialby-trial record would need to categorize the antecedent conditions, the behavioral events or states, and the consequent outcome for each recording (specifics of the record would depend on which of the items noted above was being used).

A simple approach to environmental-behavioral analysis has been summarized by Suen and Ary (1989) in their discussion of interaction matrices (pp. 25-28). Such matrices traditionally have been limited by the fact that they tend to include only consequences and, thus, ignore antecedents. They also are not typically dynamic (i.e., do not account for changes in these conditions across time or circumstance) but, rather, summarize events for an entire recording session only. On the other hand, R. D. Ray and Ray (1976) used a more comprehensive accounting that they referred to as contingency analysis. Observing children in the United States and the out-islands Bahamas, they compared teacher-student interactions to describe differences in contingency management between the two cultures. Their records included antecedent, behavior, and consequence sequences, using interval sampling techniques. As such, their analyses were limited to static comparisons between cultures and across varying contexts, such as school room interactions, playground interactions, and home environment interactions. R. D. Ray (1977) added to this approach by using a continuous recording of experimenters' use of subject-initiated trial-by-trial discrimination training procedures used by Soviet researchers. Ray also added a new analysis that tracked the temporal dynamics of antecedent, behavior, and consequence categories as they changed across time.

If environmental as well as behavioral events are to be included in the record, it will by definition be required that the chosen observation approach be able to include events that are not mutually exclusive and, therefore, should be considered as being in different domains. Thus, the issues raised in the earlier section on Methodological Implications of Mutually Exclusive and Exhaustive Taxonomies of Behavior are highly relevant.

\section{Is the taxonomy exhaustive?}

As has been noted, we developed our taxonomy by selectively searching for published exemplars to guide our operations analysis. That is, we searched the literature for as many published variations of systematic coding procedures as we could practically find. We inductively derived the common dimensions (i.e., behavior and time) from the method descriptions in all exemplars found and proceeded to define a taxonomy based on intersections of distinct subclassifications of those dimensions that emerged (i.e., cells in the matrix). What remained to be accomplished was a testing of the taxonomy with a sufficiently broad sample to evaluate how robust the taxonomy might be. We wanted to confirm that, given a generally representative sample of published research articles, we could categorize all methods using behavioral observation with our existing taxonomy. A secondary value in such an effort is a description of how such procedures are relatively applied within such a sample.

For our sample, we chose to survey one full year of publications in two divergent journals that have a strong history of publishing observational research. Thus, we 
arbitrarily selected the most recent (at the time of our research) volumes of Child Development (CD, 2006, Vol. 77) and the Journal of Applied Behavior Analysis (JABA, 2007, Vol. 40). Articles were first surveyed for discernable use of direct observational methods for data collection. Observational operations reported in the method sections of such articles were then analyzed and compared with the criteria described within the procedural cells of Tables 1-16 of the Supplemental Materials. All matches were thus categorized by the appropriate matching procedure. As the header of this section suggests, we were interested in verifying that our taxonomy was exhaustive in its categories, but we were also interested in confirming that independent observers could reliably classify research procedures using our taxonomy's operational definitions. As such, any and all questions as to appropriate categorization were resolved through discussion between the authors. This ensured that we would determine any potential need for expanding the taxonomy or clarifying operational definitions, but it also resulted in 100\% interobserver agreement. Anyone seeking to verify our classification of the procedures in these volumes could use a similar approach.

All but 6 of the 37 observational procedures found for articles in Vol. 77 (2006) of $C D$ could be classified with the taxonomy. These six exceptions reported only example phrases from field notes or frequencies from post hoc content analysis of field notes, which is a different methodology than systematic coding, and thus these studies were excluded from our review. Studies using questionnaires and surveys were also excluded from our review, since these also represent a different methodology based on self-reports. All 78 observational procedures found in Vol. 40 (2007) of $J A B A$ could be readily classified according to our taxonomy of procedures summarized in Table 2. Thus, our taxonomy was sufficiently robust to account for virtually all systematic (coding-based) observation studies found in our sample.

The utility of any taxonomy depends on the data it allows one to classify and describe. Ours is no exception. As such, our sample allows one not only to categorize any and all direct observational procedures, but also to determine the comparative or relative frequency of use for specific procedures within the subdisciplines represented by our journal selections. For $J A B A$, a high proportion of the empirical articles published in this volume used data obtained from direct observations by researchers $(91 \%$; 68 of 75). For the $C D$ journal, a much smaller proportion of empirical articles used observational procedures $(27 \% ; 34$ of 128). Note that the number of procedures found is greater than the number of articles that used systematic observation, since some articles reported use of more than one procedure in a given study.
Our data from $J A B A$ compare with $76 \%$ of the 293 empirical articles published in $J A B A$ from 1968 through 1975 reported by Kelly (1977). Thus, observational data have been used and continue to be used by the behavior analysis research community, and their use appears to be increasing. Our data from $C D$ compare with $19 \%$ of 175 empirical articles published in the 1976 volume of $C D$ (Mitchell, 1979). Thus, child development researchers continue to use observational data in about a fifth to a quarter of the articles in this major journal for their field.

Figure 2 shows that the distribution of alternative procedures published in the two journals differ in distinct ways. For $J A B A$, Procedures $3,5,7$, and 10 from summary Table 2 account for most observational procedures reported. Thus Session: Behavior Frequency/Duration (Procedure 3), Momentary Time Sampling: Behavior Yes/No Occurrence (Procedure 5), and Complete Interval Time Sampling: Behavior Yes/No Occurrence (Procedure 7) or Complete Interval Time Sampling: Behavior Frequency/Duration (Procedure 10) account for approximately $91 \%$ (71 of 78) of the alternative methods reported in 68 different articles (i.e., some articles do not report using observational methods, while others report multiple methods). For $C D$, however, the most common method was Complete Interval Time Sampling: Dominant Behavior (Procedure 8, which accounts for 12 of 37 reported procedures, or approximately $32 \%$ ). This procedure had only sparse use in $J A B A$ (approximately 4\%). Whole Session: Frequency/Duration (Procedure 3), Complete Interval Time Sampling: Behavior Yes/No Occurrence (Procedure 7), or Complete Interval Time Sampling: Behavior Frequency/Duration (Procedure 10) were also used fairly frequently in $C D$ articles (combined, they account for 12 of 37 , or approximately $32 \%$, but less frequently than in $J A B A$, where they account for 53/78, or 68\%). However, Momentary Time Sampling: Behavior Yes/No Occurrence (Procedure 5) had only one use in our $C D$ sample.

In neither journal were any variations of the continuous recording method (i.e, the variations of Procedures 14-16) much used-only $3(4 \%)$ for $J A B A$ and $2(5 \%)$ for $C D$. These procedures appear to preserve the most information from the observational records and, therefore, seem desirable. They also are the ones most aided by video/ computer-assisted observational recording, so perhaps the increasing use of technology will facilitate the use of these procedures in future research.

\section{How the taxonomy guides the modeling of procedures and designs for user interface in an expert training system}

As was noted earlier, our taxonomy was primarily developed to guide the development of software models of commonly 
used procedures for incorporation into the TTC expert training system. On the basis of the assumptions that learning to discriminate occurrences of specific behavior categories is ubiquitous across all coding procedures, the first modeled procedure incorporated for trainees in TTC was Procedure 3 in Table 2, which involves counting each behavioral category's frequency of occurrence within a given observational session. In this section, we illustrate how the specific aspects of this procedure are modeled in TTC via input and other user interface characteristics, including data display. We follow with a similar detailing of TTC's modeling of the continuous coding procedure (Procedure 16 in Table 2), since that is the procedure used by the experts who manage the development and administration of training programs delivered through TTC.

Session: behavioral frequency implementation

The specific user interface for the collection of categorical frequency counts, as well as prompts and feedback used for various adaptive levels of training services within TTC, is illustrated in Fig. 3a-d. After being alerted by a prompting "New Coding Loaded" alert dialog that signals a session's beginning (Fig. 3a), a sampling of a trainee's subsequent experiences in TTC is illustrated by the sequence of screen illustrations in Fig. 3b, c, and d. These illustrate the most heavily prompted training level-Coding Skill Level 1 (CSL1) - of the six alternative training skill levels that define the adaptive stages of TTC. Subsequent levels progressively fade the use of the illustrated prompts and feedback until, at Coding Skill Level 6 (CSL6), no prompts appear at all, and the only feedback occurs when a coding error has occurred and needs to be edited. Administrators have the option of activating a 7th Coding Skill Level that functions as a "probe" to test or certify the trainee's coding skills without any feedback whatsoever. Upon satisfying an adjustable accuracy criterion for certification across some number of codings, training on that given program is announced to be complete. Failure to meet such criteria can bring the trainee back to lower CSLs for further training as required.

Throughout the time that a countable/recordable behavioral event is occurring at training CSL1, the video depicting that event in TTC is framed in yellow as a prompt to aid in teaching trainees that a recordable event is in progress (see Fig. 3b). As Fig. 3b also illustrates, the multifaceted prompting of CSL1 includes showing beneath the video frame the codable event's categorical abbreviation, the associated keypad entry number, and the full name of the behavioral category being viewed.

When a behavioral category is functionally defined and, thus, is best coded only after the conclusion of the codable event, a short tolerance period begins, during which the video may be paused to allow a coding to be entered. Administrators may set this tolerance window to any desired duration (a duration that is recommended to become progressively shorter as training prompts are faded across successively higher adaptive skill levels in TTC as trainees develop more sophisticated coding skills). During postcategory tolerance periods, CSL1 training shows the trainee a green frame surrounding the video display to indicate that a coding period is still in progress (see Fig. 3c), and instructions beneath the video remind them that they may pause the video play to code by using the space bar. In CSL1 training, if no coding entry has occurred by the time the tolerance window elapses, a red frame appears around the video display area, and video play automatically stops to supply a feedback message. This message informs the trainee that a coding error has occurred and must be corrected (along with instructions as to how such a correction is made; see Fig. 3d).

Also illustrated in each graphic of Fig. 3 is the data entry and accumulating count panel placed just to the right of the video display. This panel indexes each frequency count any time a category is mouse-clicked or a corresponding keypad entry (denoted by bracketed numbers) is made. Because the coding procedure being trained in this expression of TTC is Session: Behavioral Frequency (Procedure 3 in Table 2), coding accuracy is determined only by event-based criteria for all time series and composite total-session IOA calculations, since time in agreement can be used only with state-based categorical coding.

\section{Continuous coding implementation}

As was just discussed regarding TTC's modeling of Procedure 3, our overall procedures taxonomy was developed to guide the TTC modeling of any or all observation/ recording procedures. In actuality, the very first procedure modeled within TTC was Continuous Coding (Procedure 16 in Table 2). This is because the trainer's (as opposed to the trainee's) codings that generate all expert or reference files used for training in TTC rely upon mutually exclusive and exhaustive behavioral taxonomies and recordings of start/stop times for all behavioral occurrences. This ensures that every frame of video used for training in TTC has one and only one corresponding behavioral state available for reference against a trainee's coding input, regardless of when that input is recorded. Thus, in TTC, if training relies upon nonexhaustive taxonomies, an other bucket category must be used in the expert's coding to identify all nonrelevant events. Importantly, administrators of all training programs to be experienced by trainees in TTC have the option of limiting training exposures to any nonexhaustive subset of the parent (and exhaustive) reference taxonomy for actual training. Any disabled 

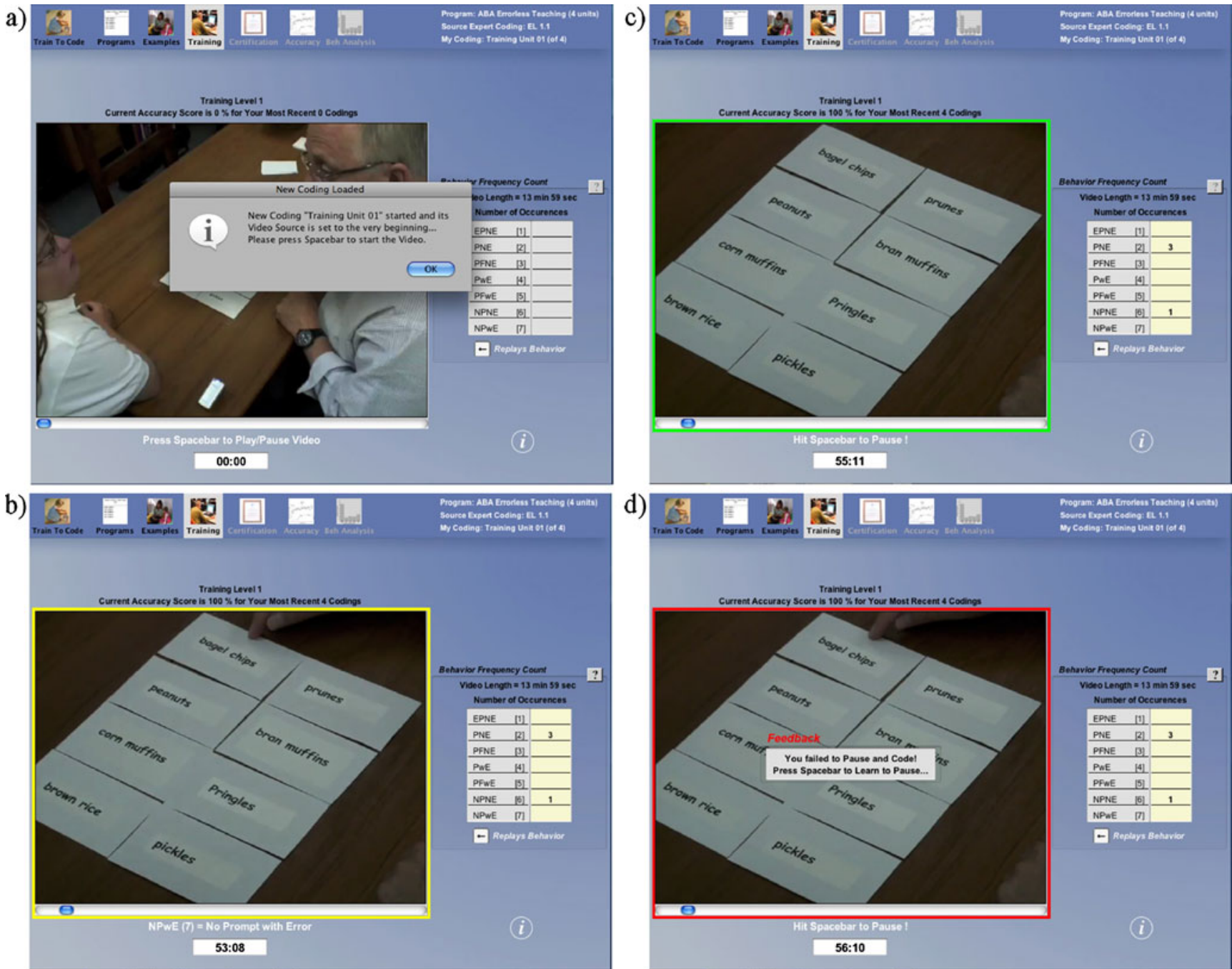

Fig. 3 Example screens showing the Session: Behavioral Frequency counting procedure (Procedure 3 in Table 2) as modeled in the TrainTo-Code software training system. The user interface, prompts, and feedback for this procedure are illustrated. a The "start coding" alert dialogue and general screen layout as seen by a trainee. $\mathbf{b}$ The yellow surround added to the video window when one of the behaviors to be coded has begun. Prior coding counts by category that were entered by the trainee are shown in the panel on the right of the video display.

categories in the exhaustive parent list combine to become a not-to-be-coded category that is prompted for purposes of trainee guidance, feedback, and accuracy calculations. As this label implies, trainees do not have to learn to actively code such excluded noncoded events (but by implication, trainees are passively doing so eventually by not entering entries for such events).

The specific interface for an expert's Continuous Coding in TTC is instructive as to the alternative interface and algorithm modeling implications of our alternative procedural categories in the taxonomy. This Continuous Coding interface is illustrated in Fig. $4 \mathrm{a}-\mathrm{c}$. It includes the video

The current time in the video is shown below the video window. $\mathbf{c}$ The green surround that is added to the video window when the behavior to be coded ends, but during the time a trainee may still stop the video and enter a code (a "tolerance window" set by the trainer). $\mathbf{d}$ The red surround of the video window signifying the error of allowing the tolerance window to elapse without coding. The video is stopped at this point, and the trainee is informed that he or she must re-view and code the past behavior before proceeding with further training

display, a coding panel allowing up to 10 category abbreviations and keypad equivalents, and a data table/ field with three columns allowing for start time, stop time, and associated behavioral category recording accumulations. Data entries accumulate as successive lines within this table/field. Note that TTC records video times from an arbitrary start time designated as zero, rather than using an absolute true time/date stamp, as might be encoded within a video source. Thus, for training purposes, it is sufficient simply to mark elapsed time from the start of the video being coded, given that coding of time-elapsed data is the only goal of the TTC system. 
Fig. 4 Example screens illustrating the Continuous Coding procedure (Procedure 16 in Table 2) as modeled by the Train-To-Code software training system. The user interface and editing options used by an expert while they are coding the video are shown. The example screens illustrate steps from the editing of an expert coding of a video that depicts a trial-by-trial teaching procedure having intertrial intervals coded as other. a Time during the video when the first trial is completed and will be coded as "Prompt with No Error" (PNE). b A person editing the already coded video data file has paused at 43:10 in the video - the start time for a trial that was coded as "No Prompt, No Error" (NPNE). The start and end times for the coded trials and intertrial intervals are shown in the field to the upper right of the video display. The trial where the video has been paused is highlighted in blue. c Editing options pop-up menu available to the person editing the expert codings, including the insertion of comments on decision criteria for this event, inserting a new additional behavior coding with that starting time, deleting the existing coded behavior with that starting time, or simply deselecting that selected behavior
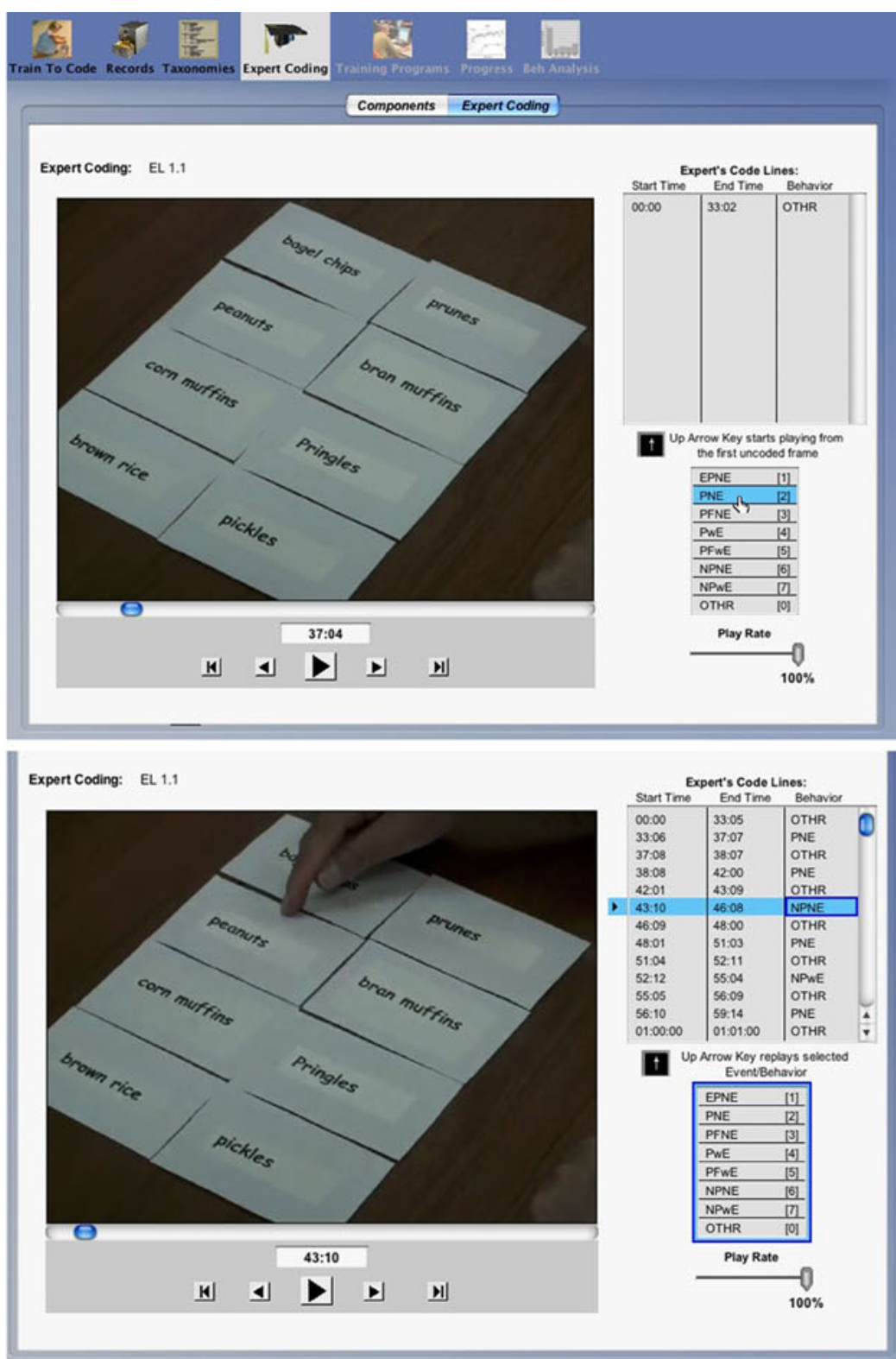

b)

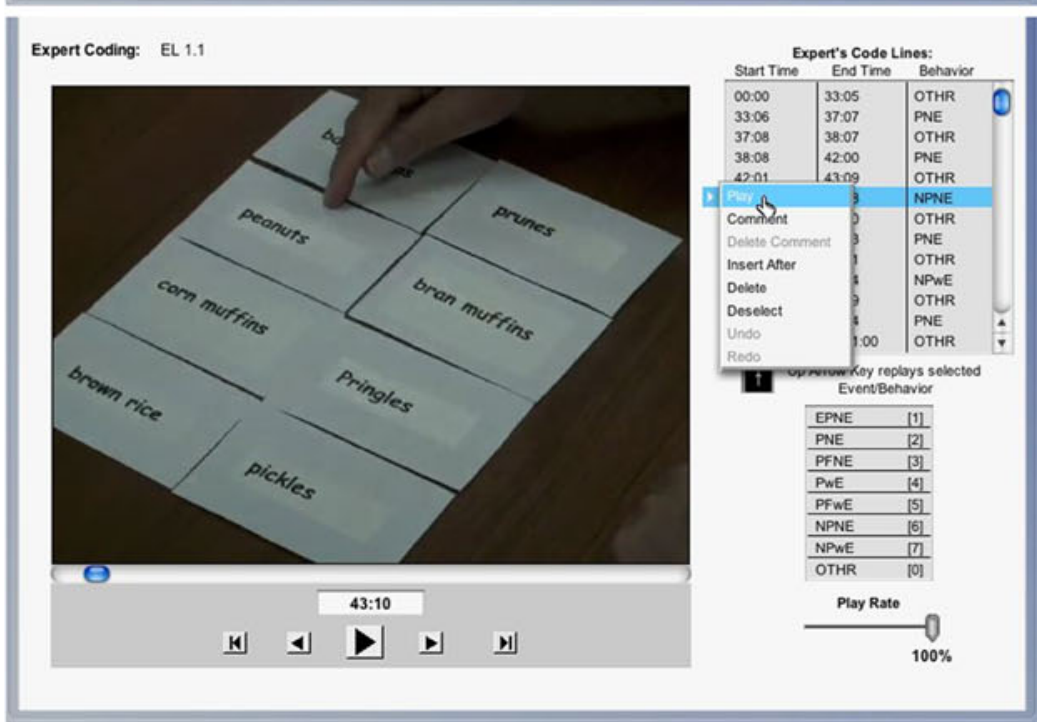


The practical result of a zero start time reference for all data listings is that, unlike our table's operationalization criteria for Continuous Coding procedures, in TTC only stop time for a current category is actually registered by the coding expert, and TTC auto-fills the derived start time for the next to-be-coded event. Typically, accurate continuous coding relies upon the unique characteristics of video-based recording and playback capabilities that include pausing video play and single-frame stepping forward or backward with respect to any video frames of special interest, such as transitions from one behavior to another or criteriachecking with regard to meeting standards required by operational definitions for classification.

Anyone who has ever watched video replays used to determine the accuracy of a referee's call of an infraction in football or another sport has had such a viewing experience. Thus, unlike the interface for trainees experiencing the Behavior Frequency counting procedure, expert coders establishing reference codings in TTC are encouraged and allowed by the Continuous Coding interface design to rely upon such video controls for precision. Likewise, editing is a dramatically different algorithm and interface experience for Continuous Coding versus Behavior Frequency counting, as we will soon see.

When the video frame corresponding to a behavioral event's stop time is found, that time stamp is recorded by entering the behavioral code describing the event just ending. As was previously noted, this data entry not only records the behavior and its corresponding stop time, but also adds one time unit (typically, one frame, or 1/30th of a second) as an auto-entered start time for the subsequent behavioral event that is about to begin. In practical terms, this recording of a current behavior's stop time while autoproducing the subsequent behavior's start time procedure is actually preferred in all cases, because coders must typically play through a transition from one behavior to another to determine that a new category of behavior has actually begun. As such, it is typically easier to step frameby-frame backward to find the exact change-over, or transition time, and then to record the behavior just ended (as opposed to the behavior just started, since that may yet to be determined by full event viewing, as with functionally defined events).

As already noted, we also quickly discovered from our modeling effort for Continuous Coding procedures in TTC that this interface and algorithm must also typically accommodate corrections and/or editing of any inputs that a coder desires, regardless of the time or sequence in their coding process that an edited entry was made. This includes editing start/stop times and/or behavioral category and, possibly, any data entry line. Importantly, behavior frequency as a procedure cannot accommodate such edits, since a simple count of, say, 5 for any given category tells you nothing about which of the five accumulated events one might be adding/subtracting. Thus, only the most recent (i.e., current) entry can be edited in that procedure, even in paper-pencil real-world recordings. Thus, it is not simply a computer-modeling restriction.

As is illustrated in Figs. 4b, c, a mouse-click selection in TTC creates a focal-frame surrounding the item selected for editing. Selection of an item simultaneously sets that item for edit and presents access to a popup menu (see Fig. 4c), allowing new item insertion, current item deletion, or even the adding of comments that can be used in training, such as the specific criteria used for resolving assignment of the selected video segment to the current category. Such editing also allows the user to change the behavioral code or the stop/start time. This is accomplished by selecting the associated item in a given data line. If a time is changed, the associated-by-inference start and/or end time is automatically corrected as well. Similarly, inserting or deleting a coded item adjusts the starting or ending time for the prior and subsequent codes as well.

\section{Conclusion}

An initial literature survey that was quite broadly focused generated an operations analysis of as many alternative reported procedures for collecting data through systematic observation as we could find. From this operations analysis, we generated an organizational scheme, or procedural taxonomy, for systematically classifying, comparing, and contrasting those procedures. We subsequently conducted a more targeted survey of two disparate journal volumes to confirm the inclusiveness of that taxonomy. We found that $100 \%$ of published articles incorporating observational methods with systemic coding for data collection defined by the present taxonomy were classifiable. We found 6 $(5 \%)$ articles that used only field notes as sources, which we never intended to incorporate as coding procedures to be modeled or taught. They used direct observation, but not systematic coding schemes. We thus are encouraged that our taxonomy is robust and will apply to the use and teaching of criteria for defining systematic data recording procedures likely to be used in direct observational research settings.

Concurrent with our survey was our development of TTC as a means for automating the training of observers who are typically tasked with collecting the data in such observational research. This project was yet another convergent affirmation of our alternative procedural specifications; that is, we found our procedure descriptions sufficiently specific to guide each stage of design decision and algorithm development, not only for training, but also for the expert system specifications and for evaluating IOA 
between trainee and expert. Our taxonomy specifications also guided our alternative data analysis designs, both for error analysis and for summarizing the video source events. Thus far, our development efforts on TTC have confirmed the adequacy of all of our original specifications.

Perhaps more important, the discipline imposed by developing computer software has forced us to systematize an important and complex family of methods that is in near disarray. Incomplete reports of procedures used are common, and when complete reports do exist, a consistent vocabulary for naming the procedures is lacking. We feel that the present taxonomy brings a much needed order to behavioral observation research. We also believe that we have confirmed through our sample survey that behavioral observation is a very important dimension of behavioral research methods.

\section{References}

Altmann, J. (1974). Observational study of behavior: Sampling methods. Behaviour, 49, 227-267.

Astley, C. A., Smith, O. A., Ray, R. D., Golanov, E. V., Chesney, M., Chalyan, V. G., ... Bowden, D. M. (1991). Integrating behavior and cardiovascular responses: The code. American Journal of Physiology: Regulatory, Integrative, and Comparative Physiology, 261, R172-R181.

Baer, D. M. (1977). Perhaps it would be better not to know everything. Journal of Applied Behavior Analysis, 10, 167-172.

Bakeman, R., \& Gottman, J. M. (1987). Applying observational methods: A systematic view. In J. D. Osofsky (Ed.), Handbook of infant development (pp. 818-854). New York: Wiley.

Bakeman, R., \& Gottman, J. M. (1997). Observing interaction: An introduction to sequential analysis (2nd ed.). Cambridge: Cambridge University Press.

Boykin, R. A., \& Nelson, R. O. (1981). The effect of instructions and calculation procedures on observers' accuracy, agreement, and calculation correctness. Journal of Applied Behavior Analysis, 14, 479-489.

Campbell, J. T. (1976). Psychometric theory. In M. D. Dunnette (Ed.), Handbook of industrial and organizational psychology (pp. 185222). Chicago: Rand McdNally.

Cohen, J. (1960). A coefficient of agreement for nominal scales. Educational and Psychological Measurement, 20, 37-46.

Collins, J. J., \& Stewart, I. N. (1993). Coupled nonlinear oscillators and the symmetries of animal gaits. Journal of Nonlinear Science, 3, 349-392.

Cone, J. D. (1982). Validity of direct observation assessment procedures. In D. P. Hartmann (Ed.), Using observers to study behavior (New Directions for Methodology of Social and Behavioral Science, Vol. 14, pp. 67-79). San Francisco: JosseyBass.

Cronbach, L. S., Gleser, G. C., Nanda, H., \& Rajaratnam, N. (1972). The dependability of behavioral mesurements. New York: Wiley.

Cunado, D., Nixon, M. S., \& Carter, J. N. (2003). Automatic extraction and description of human gait models for recognition purposes. Computer Vision and Image Understanding, 90, 1-41.

Friman, P. C. (2009). Behavior assessment. In D. H. Barlow, M. K. Nock, \& M. Herson (Eds.), Single case experimental designs: Strategies for studying behavior change (3rd ed., pp. 99-134). Boston: Pearson.
Grove, W. M., Andreasen, N. C., McDonald-Scott, P., Keller, M. B., \& Shapiro, R. W. (1981). Reliability studies of psychiatric diagnosis. Archives of General Psychiatry, 38, 408-413.

Guggenmoos-Holzmann, I. (1993). How reliable are chance-corrected measures of agreement? Statistics in Medicine, 12, 2191-2205.

Hartmann, D. P. (1977). Considerations in the use of interobserver reliability estimates. Journal of Applied Behavior Analysis, 10, 103-116.

Hartmann, D. P., \& Wood, D. D. (1992). Observational methods. In A. S. Bellack, M. Hersen, \& A. E. Akzdin (Eds.), International handbook of behavior modification and therapy (pp. 107-138). New York: Plenum.

Hawkins, A., Sharpe, T. L., \& Ray, R. D. (1994). Toward instructional process measurability: An interbehavioral field systems perspective. In R. Gardner, D. Sainatok, T. Cooper, T. Heron, W. Heward, J. Eshleman, \& T. Grossi (Eds.), Behavior analysis in education: Focus on measurably superior instruction. (pp. 241-255). Pacific Grove, CA: Brooks/Cole.

Herrnstein, R. J. (1961). Relative and absolute strength of response as a function of frequency of reinforcement. Journal of the Experimental Analysis of Behavior, 4, 267-272.

Holland, J. G., \& Skinner, B. F. (1961). The analysis of behavior: A program for self-instruction. New York: McGraw-Hill.

House, A. E., Farber, J., \& Nier, L. L. (1983). Differences in computational accuracy and speed of calculation between three measures of interobserver agreement. Child Study Journal, 13, 95-201.

Kelleher, R. T., \& Gollub, L. R. (1962). A review of positive conditioned reinforcement. Journal of the Experimental Analysis of Behavior, 5, 543-597.

Kelly, M. B. (1977). A review of the observational data-collection and reliability procedures reported in The Journal of Applied Behavior Analysis. Journal of Applied Behavior Analysis, 10, 97-101.

Lee, M. D., \& Del Fabbro, P. H. (2002). A Bayesian coefficient of agreement for binary decisions. Retrieved from http://www. psychology.adelaide.edu.au/members/staff/michaellee/homepage/ bayeskappa.pdf.

Mann, J., Have, T. T., Plunkett, J. W., \& Meisels, S. J. (1991). Time sampling: A methodological critique. Child Development, 62, 227-241.

Martin, P., \& Bateson, P. (1993). Measuring behavior: An introductory guide. London: Cambridge University Press.

Mitchell, S. K. (1979). Interobserver agreement, reliability, and generalizability of data collected in observational studies. Psychological Bulletin, 86, 376-390.

Mudford, O. C., Martin, N. T., Hui, J. K. Y., \& Taylor, S. A. (2009). Assessing observer accuracy in continuous recording of rate and duration: Three algorithms compared. Journal of Applied Behavior Analysis, 42, 527-539.

Mudford, O. C., Taylor, S. A., \& Martin, N. T. (2009). Continuous recording and interobserver agreement algorithms reported in the Journal of Applied Behavior Analysis (1995-2005). Journal of Applied Behavior Analysis, 42, 165-169.

Purton, A. C. (1978). Ethological categories of behavior and some consequences of their conflation. Animal Behavior, 26, 653670.

Ray, R. D. (1977). Physiological-behavioral coupling research in the Soviet Science of Higher Nervous Activity: A visitation report. Pavlovian Journal of Biological Sciences, 12, 41-50.

Ray, R. D., \& Delprato, D. J. (1989). Behavioral systems analysis: Methodological strategies and tactics. Behavioral Science, 34, 81-127.

Ray, R. D., \& Ray, M. R. (1976). A systems approach to behavior II: The ecological description and analysis of human behavior dynamics. Psychological Record, 26, 147-180. 
Ray, J. M., \& Ray, R. D. (2008). Train-to-code: An adaptive expert system for training systematic observation and coding skills. Behavior Research Methods, 40, 673-693.

Sanford, E. C. (1888). Personal equation. American Journal of Psychology, II, 3-38.

Sharpe, T., \& Koperwas, J. (2003). Behavioral and sequential analysis: Principles and practice. Thousand Oaks, CA: Sage.

Skinner, B. F. (1938). The behavior of organisms. New York: Appleton-Century-Crofts.

Spitznagel, E. L., \& Helzer, J. E. (1985). A proposed solution to the base rate problem in the kappa statistic. Archives of General Psychiatry, 42, 725-728.

Suen, H. K., \& Ary, D. (1989). Analyzing quantitative behavioural observation data. Hillsdale, NJ: Erlbaum.

Suen, H. K., \& Lee, P. S. C. (1985). Effects of the use of percentage agreement on behavioral observation reliabilities: A reassessment. Journal of Psychopathology and Behavioral Assessment, 7, 221-234.
Terrace, H. S. (1963). Discrimination learning with and without "errors." Journal of the Experimental Analysis of Behavior, 6, 1-27.

Thorndike, E. L. (1898). Animal intelligence: An experimental study of the associative processes in animals. Psychological Monographs, 2(4), Whole No. 8.

Thorndike, E. L. (1899). A reply to "The nature of animal intelligence and the methods of investigating it." Psychological Review, 6, 412-420.

Verplanck, W. S. (1957) A glossary of terms. Psychological Review, 64 (Suppl.), 42 and i.

Verplanck, W. S. (1996). From 1924 to 1996 and into the future: Operation analytic behaviorism. Mexican Journal of Behavior Analysis, 22, 19-60.

Vollmer, T. R., Soloman, K. N., \& St. Peter Pipkin, C. (2008). Practical implications of data reliability and treatment integrity monitoring. Behavior Analysis in Practice, 1, 4-11.

Wallace, B., \& Ross, A. J. (2006). Beyond human error: Taxonomies and safety science. Boca Raton, FL: CRC Press. 